Mots. Les langages du politique

\title{
Les emprunts discursifs entre politique et publicité. Des échanges inégaux
}

Denis Barbet

\section{(2) OpenEdition \\ Journals}

Édition électronique

URL : http://journals.openedition.org/mots/20590

DOI : $10.4000 /$ mots. 20590

ISSN : 1960-6001

Éditeur

ENS Éditions

\section{Édition imprimée}

Date de publication : 1 mai 2012

Pagination : 15-30

ISBN : 978-2-84788-356-5

ISSN : 0243-6450

\section{Référence électronique}

Denis Barbet, «Les emprunts discursifs entre politique et publicité. Des échanges inégaux 》, Mots. Les langages du politique [En ligne], 98 | 2012, mis en ligne le 26 novembre 2012, consulté le 17 mai 2019. URL : http://journals.openedition.org/mots/20590 ; DOI : 10.4000/mots.20590

\section{(c) ENS Éditions}




\section{Les emprunts discursifs entre politique et publicité. Des échanges inégaux}

Le monde politique, comme indiqué dans la présentation du dossier, emprunte très largement à la publicité commerciale ses méthodes et techniques, mais moins souvent ses mots. S'il arrive que des locuteurs politiques s'approprient des éléments de discours issus de l'univers de la publicité ou des marques, les cas restent assez limités. Peut-être les politiques rechignent-ils à puiser massivement dans le répertoire marchand par crainte de rendre trivial leur objet? Il est sans doute plus valorisant de nourrir un discours politique de références réputées plus nobles, par exemple historiques ou littéraires. En tout cas, les importations de ce genre exigent que les expressions publicitaires recyclées appartiennent à une culture assurément partagée par les publics visés et bénéficient d'une notoriété suffisante. Leur avantage, comme pour les emprunts au lexique sportif (Barbet, 2007), est alors de parler au plus grand nombre.

\section{De la publicité vers la politique}

La référence à la publicité concerne le plus souvent des slogans : ainsi, dans son discours de clôture des « universités d'été » du Front national à Nice (11 septembre 2011), Marine Le Pen reprend la devise de la marque Sony pour dénoncer la collusion de l' «UMPS» et défendre le monopole du FN dans la «vraie lutte contre l'immigration »: «L'interdiction de renvoyer dans leur pays les étrangers criminels, les socialistes l'avaient rêvé, Sarkozy l'a fait ! ${ }^{\prime »}$

D’autres slogans à succès ont sans doute alimenté, de façon fugitive ou volatile, des conversations politiques ordinaires, dont on peut trouver des traces sur des sites militants : par exemple, un opposant à Nicolas Sarkozy détourne en mars 2010 la double injonction «Votez... éliminez »² (celle de Vittel était «Buvez-Eliminez»), et un partisan d’A. Montebourg titre en janvier 2011

1. Site 1. Le slogan commercial «J'en ai rêvé, Sony l'a fait», désormais ancré dans la mémoire collective, date de la fin des années 1980.

2. Site 2 .

Université de Lyon, Institut d'études politiques, CNRS (Triangle)

denis.barbet@sciencespo-lyon.fr

Mots. Les langages du politique $\mathrm{n}^{\circ} 98$ mars $2012 \bullet 15$ 
son post «Avec Arnaud Montebourg, je positive!»3 (l'original, avec «Carrefour», a été créé en 1988).

Les hommes politiques font parfois appel à des marques commerciales, en s'appuyant plus ou moins consciemment sur la notoriété d'un produit. L'exemple de «Kärcher» vient à l'esprit : le 20 juin 2005, au lendemain de la mort par balle d'un jeune garçon qui lavait la voiture de son père au bas d'un immeuble de la Courneuve, le ministre de l'Intérieur, alors Nicolas Sarkozy, rend visite à la famille de la victime et lui annonce le prochain «nettoyage de la cité au Kärcher ». Il justifie ensuite ses propos dans un échange avec les jeunes du quartier : "Le terme “nettoyer au Kärcher” est le terme qui s’impose, parce qu’il faut nettoyer cela4. " S'appropriant les qualités supposées des nettoyeurs à haute pression - dont la marque est consacrée au point d'avoir par antonomase, à l'instar de Frigidaire, donné son nom aux appareils de ce type -, le prétendant à l'Élysée cherche à montrer sa puissance et son efficacité dans la lutte contre la violence en banlieue. Si l'expression entraîne à l'époque une vive polémique, nous ne retiendrons ici que la réaction, près de deux ans plus tard, du groupe Kärcher-France. Une mise au point dans la presse 5 lui offre le double avantage de lever "tout malentendu » et de vanter les mérites de ses marchandises et de leur distribution. La société proteste contre les abus répétés de son nom, éloignés «de [son] univers de communication habituel», et précise qu'elle ne peut se reconnaître dans les amalgames auxquels sa marque est associée. Ses dirigeants écrivent notamment aux candidats à la présidentielle pour rappeler l'exclusivité des droits d'une marque déposée. La reprise de l'expression en 2010 par Fadela Amara, secrétaire d’État chargée de la Politique de la Ville (Le Progrès de Lyon, 11 janvier 2010), conduit l'enseigne à rappeler sèchement le gouvernement au respect du droit des marques («n'employer le terme Kärcher $®$, [qui] n’est pas un nom commun, que pour désigner ses produits »), et à se retirer notamment de l'opération de nettoyage de la façade du palais de Chaillot ${ }^{6}$. Au-delà du fond politique des discours, on peut penser que leur effet sur les achats des consommateurs est très improbable et que l'entreprise a bénéficié en l'occurrence d'une promotion gratuite inespérée.

Les messages sont parfois pris dans de curieux chassés-croisés. Si le Parti communiste, par exemple, fait l'objet d'une captation publicitaire fondée sur l'homonymie de siglaison entre Parti Communiste et Personal Computer (une affiche de Commodore Computer porte le slogan : «Le PC se démocratise», afin de montrer que les produits sont abordables7), ce parti peut aussi, en 2000, parodier (Bonhomme, 2006 et dans ce dossier) certaines annonces de

\footnotetext{
3. Site 3.

4. JT, France 2, 29 juin 2005 . Vidéo : site 4.

5. Le Monde, 16 mars 2007.

6. Site 5; «La facture de Kärcher », Le Canard enchaîné, 10 février 2010.

7. Reproduite par Cathelat et Ebguy, 1988, p.167.
} 
la marque Benetton pour une campagne contre les inégalités. De même, quand Nicolas Sarkozy prononce un nom de marque, les journalistes en utilisent un autre pour désigner l'énergique prétendant à l'Élysée : «le lapin Duracell de la politique », en référence à une publicité qui présente un jouet continuant à frapper sur son tambour, alors que les autres ont depuis longtemps épuisé les piles de la concurrence...

Mais malgré ces différents exemples, les dires circulant en sens inverse sont les plus répandus.

\section{De la politique vers la publicité}

\section{Usages du passé}

Les publicitaires tendent en effet à annexer fréquemment des événements politiques, passés ou présents. Dans le premier cas sont sollicités des faits historiques marquants - comme «la révolution» ou Mai 68 - ou des «grands hommes» (Chauveau, Veyrat-Masson, 2004). L'objectif est de conférer aux produits concernés la renommée et la profondeur de l'histoire et de la tradition. Le constructeur Citroën s'est à ce titre durablement servi du prestige du fondateur de la Ve République. Non seulement la marque s'enorgueillit, aux débuts du Régime, d'être celle des présidents, mais elle se targue d'avoir permis au général de Gaulle d'échapper à l'attentat du Petit-Clamart en août 1962. Ce «miracle» trouve son explication commerciale dans la fiabilité du véhicule à bord duquel il circulait : la «mythique» DS 19. Citroën en tirera d'ailleurs le slogan : «Ce que cette marque a pu faire pour un général, elle peut le faire pour tout le monde ${ }^{8}$. Cette labellisation historique se retrouve encore dans un spot télévisé de l'entreprise, en 2009, à côté de nouveaux signes de modernité (la Formule 1) : le chef de la Résistance est convoqué, qui envoie de la BBC cet «appel» aux Français : «Je voudrais une DS »9.

Le fabricant de fosses septiques Eparcyl s'est distingué, en février 1999, dans la même rubrique de référence aux grands ancêtres politiques, de gauche cette fois. Le notoire slogan de François Mitterrand en 1981 est détourné avec humour: «Eparcyl, la fosse tranquille ${ }^{10}$... Des phrases célèbres ou des scènes cultes de la vie politique sont susceptibles d'être absorbées par la publicité. C'est le cas du «Yes we can » de Barack Obama (par Chevrolet, pourvanter une offre de TVA gratuite) ou de l'«Au revoir » de Valéry Giscard d'Estaing après sa défaite présidentielle, par le 118-218, en avril 2007 (Héraud, 2007).

Dans son étude classique de l'affiche de Volkswagen pour la Golf en

\footnotetext{
8. Cité par Chauveau et Veyrat-Masson, 2004, p.127.

9. Site 6.

10. Visuel sur le site 7 .
} 
1991-1992, où les outils-icônes de la panne réfèrent métaphoriquement à l'effondrement du système soviétique, Emmanuël Souchier (1992, p.50) décelait avec perspicacité un renversement de tendances dans les rapports entre la publicité et la politique, en distinguant trois phases. Après avoir durablement gommé la politique du contenu de leurs messages, les professionnels de la publicité ont investi la sphère politique, dans le contexte économique faste et plutôt mobilisé politiquement des années 1960-1970. Cette période d'essor du marketing politique leur a permis de mettre leur savoir-faire mercatique au service des professionnels de la politique, en contribuant à promouvoir partis et candidats comme des marchandises et à dépolitiser leur discours. Dans un troisième temps, à partir des années 1980-1990, marquées par la crise et un certain retrait du politique, les publicitaires n'hésitent plus à s'approprier la substance politique elle-même et les discours des acteurs, en les récupérant à des fins commerciales. Cette évolution, qui s'est poursuivie et nettement amplifiée depuis, est en réalité amorcée antérieurement ${ }^{11}$.

\section{L'actualité électorale au rebond}

Quand les enseignes sont inspirées par le temps politique présent, elles se nourrissent très largement d'objets électoraux. Les annonceurs s'adossent alors, dans une logique de rebond ou d'embuscade (on parle d'ambush marketing : Bénaroya, 2010), à l'actualité des élections. Cette tendance apparaît dans la presse des lendemains de scrutins nationaux, qui fournit dès les années 1970 son lot de messages publicitaires, insérés en pleine page, mêlés au traitement de l'événement. Pour ne retenir que quelques exemples, à partir d'un rapide sondage dans Le Monde et Libération :

- un cabinet immobilier présente, après le premier tour des législatives de 1973, la photographie de son nouvel immeuble parisien avec la mention : «Nous avons gagné 124 sièges!... pour asseoir nos clients et nos collaborateurs [...]» (Le Monde, 6 mars 1973);

- l'éditeur Albin Michel introduit ses meilleures ventes de livres, à la une, par le texte à double jeu de mots : «Élus par les lecteurs au $1^{\mathrm{er}}$ tour » (Le Monde, 21 mars 1978);

- IKEA se gausse des battus dans une pleine page de Libération, le 17 mars 1986 : «Tous ceux qui ont perdu leur siège hier peuvent le récupérer aujourd'hui chez IKEA [...] Et pour ceux qui se sont vraiment faits [sic] étaler, nous avons aussi de très beaux canapés »;

- dans le même quotidien (p.22), les stages Vitaline de Luchon publient

11. Dans les années 1960, marquées par l'usage gaullien des référendums, Simca innove en demandant « un oui franc et massif» pour son modèle 1300 et Darty propose son «Programme commun » dès les années 1970 (Albouy, 1994, p.157, note 18). 
les photos de quatre vedettes politiques visiblement fatiguées, avec le commentaire : «Épuisant, non ?», qui se termine par «Après la campagne, la montagne» et une invitation à contacter la société12.

Les fondations de la captation publicitaire sont posées : le produit est un candidat, et souvent l'élu; les acteurs politiques, consommateurs virtuels, commencent à servir d'instruments de promotion commerciale. Leur image, réelle ou déformée ${ }^{13}$, est utilisée dans des messages qui les interpellent en les moquant à l'occasion. Les publicitaires utilisent la polysémie ou le calembour et jouent sur l'agenda des préoccupations sociales, pour recycler l'air - et les paroles - du temps, "se décaler» par rapport à des situations ou messages réels, dont une partie des citoyens peut se lasser, des promesses électorales en particulier. Ainsi, la promotion de la Golf Memphis profite dès 1981 du contexte de campagne présidentielle pour se démarquer, par ses affiches $4 \times 3$, de celles des candidats, avec le message: "Ça ne porte pas de cravate, ça ne sourit pas, mais au moins ça tient ses promesses. Volkswagen. C'est pourtant facile de ne pas se tromper ${ }^{14}$.

Véritables «éponges de l'actualité », les agences publicitaires ont depuis longtemps, dans leurs emprunts politiques, dépassé l'ellipse des personnes ou la simple allusion. Elles n'hésitent plus à travestir les acteurs (en les déguisant, en utilisant des sosies) ou à déformer leurs propos (en leur prêtant les leurs). Plus ou moins subtils, les messages usent désormais d'un ton plus direct, impertinent et sarcastique ${ }^{15}$. Cette évolution est associée à la diversification des supports de communication. Les campagnes commerciales élargissent leur palette médiatique et leurs cibles, elles reposent désormais sur un "plan média multicanal », qui associe Internet à plusieurs vecteurs traditionnels. Si l'ampleur des opérations dépend de leur coût, l'adossement à l'existant se révèle précisément moins cher que la création. Le travail des agences est aussi modifié par le développement du marketing «viral», dont le buzz est une forme particulière, destinée à faire du «bruit» (ou du ramdam) pour se faire connaître. Les messages commerciaux sont désormais propagés - à l’instar des virus informatiques - par les usagers eux-mêmes, par la voie électronique et

12. Pour d'autres exemples, voir notamment Le Monde, 11 mars et 16 mars 1978 (CII Honeywell Bull); 18 mars 1986 : une société proclame ses produits «élus à l'unanimité »; un conseil en recrutement remercie tous ceux qui l'ont «élu » et déclare : «Voici 25 ans que nous faisons cohabiter les Français. Vaste programme !». Pour la présidentielle de 1988, voir Libération, 25 avril 1988 (trois annonces vantent des produits - notamment IKEA - en faisant référence à l'élection) et 9 mai 1988 (la Fnac s’adresse au réélu : «Cher Président, n’oubliez pas la musique dans votre victoire »). Dans Le Monde du 10 mai 1988, l'École supérieure de commerce de Dijon fait la leçon aux candidats : « $46 \%, 54 \%$ !.... Monsieur Chirac, Monsieur Mitterrand, chez nous vous auriez appris à être de vrais rassembleurs!»

13. À l'occasion des législatives de 1993, par exemple, la campagne «Oui à la France qui rit», de la marque de vêtements Waikiki, «singe», dans tous les sens du terme, plusieurs personnalités politiques en leur donnant une apparence simiesque (Albouy, 1994, p. 157).

14. Voir également la publicité pour le «candidat» Speed Burger (Bénaroya, 2010).

15. Voir les sites 8 et 9 , orientés sur les «nouveautés». 
les réseaux de connaissance. Ce procédé, qui offre l'avantage de diffuser très rapidement et gratuitement une communication en phase avec l'actualité à des publics ciblés, a été utilisé par plusieurs campagnes publicitaires mentionnées ci-après. La démultiplication et la prolifération de ces messages portent des risques de banalisation qui contribuent à expliquer que les emprunts discursifs de la publicité à la politique se diversifient mais tendent aussi à devenir plus audacieux. Remarquons également que l'élection, «traduction démocratique » de la concurrence à laquelle se livrent les acteurs pour le pouvoir politique, ne peut laisser insensibles les agences de publicité, confrontées elles-mêmes à une rude compétition commerciale ${ }^{16}$. Focalisées sur cet enjeu, elles cherchent à se singulariser (Charaudeau, 2005, p. 237) dans la promotion des marques de leurs clients et rivalisent d'« originalité » dans l'exploitation de l'actualité, pour se distinguer de la concurrence et capter l'attention des destinataires.

\section{Procédés de détournement}

Les modalités selon lesquelles les publicitaires s'emparent de l'objet électoral sont variables. Le cas particulier de l'interpellation doit être distingué. L'opportunité qu'offre la conjoncture est alors saisie pour sensibiliser l'opinion publique et faire pression sur les politiques. L'annonceur peut être sollicité par un défenseur de cause : l'association Aides contre le Sida fait appel à l'agence TBWA et publie des portraits des candidats à la présidentielle de 2007, avec leur accord, pour lutter contre les discriminations envers les malades : «Voteriez-vous pour moi si j'étais séropositif(ve) ?» (Maudieu, 2007) Plus généralement, les publicitaires, qui tendent à occulter la dimension marchande de leur démarche, livrent aujourd'hui volontiers les produits dans un emballage «citoyen " (ou « éthique », ou encore «militant»). Désireuses d'accroître la légitimité de leur prise de parole (Granier, 2008, p. 10), les entreprises sont ainsi enclines à se substituer à l'autorité publique dans la diffusion de messages d'intérêt général aux citoyens-consommateurs, pour les mobiliser ou répondre à des «attentes» sociétales : prévention sanitaire, protection des aliments, de l'environnement, commerce équitable, défense des droits de l'homme, etc. ${ }^{17}$ Par exemple, la marque de vêtements de sport Patagonia invite les électeurs à tenir compte des engagements des concurrents de 2007 en matière environnementale, comme elle l'avait fait trois ans auparavant aux États-Unis, en appelant implicitement à voter pour le démocrate John Kerry (Bénaroya, 2010). Les fermiers du bio (Danone et Stonyfield), dans le cadre de leur opération «Les

16. Par exemple, pour le visuel Thomson traité plus loin, le responsable du budget de l'opération indique : «Il fallait faire un coup, sur un marché très concurrentiel, sans y consacrer un gros budget. Il fallait rebondir sur l'actualité, or la présidentielle sur Internet était déjà perçue comme un gros enjeu. » (Mathieu Galloux, cité par Les Échos, 12 avril 2007)

17. Exemples sur le site 10. 
deux vaches » en 2007, mettent à disposition des clients 600000 cartes postales humoristiques à expédier aux candidats, que les bovins interrogent sur leur sincérité écologique ${ }^{18}$. L'année suivante, pour les municipales, les deux bêtes sont converties en manifestantes qui portent des banderoles réclamant du bio dans les cantines scolaires (liberation.fr, 2 mai 2008).

Dans la plupart des cas, les enseignes recyclent le lexique politique en accommodant des discours récurrents tenus en période électorale et en jouant sur les codes des candidats ou des commentateurs. La société d'informatique IC simule par exemple l'annonce des résultats et propose «en exclusivité, les meilleurs chiffres du $2^{\mathrm{e}}$ tour $»$ : ceux obtenus en réalité par ses propres produits, accompagnés de leurs prix, deux doubles drapeaux tricolores croisés officialisant l'information (Libération, 9 mai 1995, au lendemain de la présidentielle).

Mais parmi les procédés les plus classiques, qui perdurent, l'injonction et l'invite électorales restent déterminantes. Il s'agit toujours plus ou moins de faire «élire» ou «plébisciter» le «produit-candidat» par les usagers. Dans la lignée d'expériences déjà citées, on retiendra des opérations publicitaires qui présentent en 2007 un concurrent ou un parti fictif incarnant la marque ou reprenant ses engagements commerciaux ${ }^{19}$ :

- un vendeur de confiserie appelle ses clients à soutenir son produit («Votez Friskman, le seul candidat [du parti de la fraîcheur] qui n'a pas peur d'ouvrir la bouche»), sur le site www. louvriren2007.com. Plusieurs visuels repris sur Dailymotion pastichent des meetings ou des allocutions présidentielle ${ }^{20}$;

- un distributeur multimédia (Connexion) recommande en mars le vote UMPP (Union du Multimédia à Petits Prix), en imitant des slogans : «Unis, nous vaincrons» ou «Un PC pour tous».

Dans d'autres cas, les enseignes se font tout simplement élire, comme Danone, qui tente un nouveau performatif : "On vote tous pour Danette» (janvier-mars 2007), ou les conserves d'Aucy, qui font plébisciter leur nouveau produit «saveur de l'année».

Le filon de l'injonction civique, répétée à l'envi en période électorale, est également exploité, notamment par l'Ensemble orchestral de Paris, qui entend mobiliser son public, dans un encart publicitaire : «Pas d'abstention : choisissez l'abonnement ${ }^{21}$ ».

Le champ lexical de l'élection investi par les «créatifs» tend, on le voit, à s'élargir au-delà de la simple invite. Les prétendus candidats sont désormais dotés des attributs de l'acteur politique : sigles partisans inventés, programmes et slogans transformés, QG de «campagne» fictifs, faux «adhérents», etc. La campagne du vendeur de glaces Ben \& Jerry's (agence Magic Garden) offre à

18. Site 11.

19. Pour les exemples qui suivent et d'autres cas, voir Bénaroya, 2010, p. 4 ; Héraud, 2007.

20. Site 12.

21. Le Monde TV et radio, mai-juin 2007. 
ce titre un bel amalgame : en mars 2007 est lancée, en teasing, une série d'affiches annonçant une candidature de dernière minute, dont l'identité est révélée en avril sur un site Internet (www.lautrecandidate.com) : il s'agit encore d'une $v^{\text {vache }}{ }^{22}$, Woody, ceinte d'une écharpe tricolore : «La première candidate... de poids, militant pour une France gourmande... qui promet plus d'un pot... instaure le pré à taux zéro ». Le site communique les informations de sa campagne, relayées par du Street Marketing et par plusieurs médias. Le 17 avril, ses «partisans » se regroupent pour une "grande journée de mobilisation» : flyers et animations de rue annoncent des dégustations gratuites et le soir, un cortège se rend à l'Élysée (-Montmartre...). Le tout «enrobé» dans un message « citoyen »: inciter la population à peser lors des élections, comme la marque l'avait déjà fait aux États-Unis en défendant les petites coopératives laitières familiales 23 .

Les messages s'appuient souvent sur des expressions électorales courantes, qu'il suffit de dévier de leur sens initial: Mercedes-Benz, par exemple, invite le consommateur à saisir son offre spéciale, pendant la campagne des régionales en mars 2010, en affichant qu' «il n'y aura pas de deuxième tour» (situation au demeurant peu probable dans ce type de scrutin). Le syntagme «vote utile», ambigu mais couramment employé, est également retraité. Sa signification reste sans doute assez floue pour une partie de la population, malgré les constants rappels au souvenir du 21 avril 2002, et sa modalisation adjectivale (qu'est-ce qui est «utile»?), tout comme sa définition, peut être contestée. Circulant dans la controverse, la formule (Krieg-Planque, 2009) se prête particulièrement à la récupération publicitaire. La chaîne de grandes surfaces Intermarché-Écomarché lance ainsi en septembre 2009 (entre les Européennes de juin et les régionales de mars 2010), une campagne "vote utile», qui fournit un titre à son magazine commercial24. L'expression est en outre censée donner une image interactive à l'enseigne, saluée dans le milieu professionnel comme "une concrétisation du "pouvoir" rendu aux consommateurs $25 »$. Elle est d'ailleurs reprise en janvier 2012 (sous la forme «votez utilitaire») par Citroën.

Les annonceurs peuvent jouer enfin sur l'homonymie et le suspens électoral, comme l'agence DDB Paris, qui profite de la conjoncture de 2007 pour proposer à la marque de Camembert «Président», au nom prédestiné, un dispositif multimédia. Le teasing entre les deux tours se conclut le 6 mai, soir de l'élection, par la révélation télévisée du «visage» du nouvel élu : un fromage, «le nouveau Président», présenté dans un conditionnement rénové...

Tous les scrutins nationaux sont concernés par cette captation publicitaire.

22. L’animal symbolisant la «campagne», dans un troisième sens ni politique ni publicitaire...

23. Cf. Les Échos, 12 avril 2007 et site 13.

24. Cf. Site 14. Un professionnel de l'hébergement de sites Web, Wiki-LWS, a créé un domaine «votezutile.com».

25. Comme l'indique un participant aux journées du magazine de la distribution alimentaire Linéaires (site 15). 
Pourtant la présidentielle, échéance majeure dans la vie politique française et la plus participative, est la plus prisée, surtout quand elle suscite un certain engouement citoyen, comme en 2007. Cette période a été très productive en détournements commerciaux. Plusieurs annonceurs s'y sont distingués dans les variantes de l'art du « rebond » événementiel.

\section{7 en «campagnes »}

Plusieurs opérations combinant les procédés déjà évoqués, synthétisant leur évolution ou révélant de nouvelles orientations publicitaires méritent d’être retenues :

- La campagne multimédia d'IKEA «Oui au changement», conçue par l'agence La Chose entre octobre 2006 et mai 2007, illustre assez bien le détournement du discours politique. Sa déclinaison d'affiches s'inscrit dans un contexte où les prétendants à la fonction suprême convoitent les thèmes du «changement $^{26}$ et de la «nouveauté». L'entreprise, qui n'en est pas à son premier essai parodique, expose son identité commerciale sur un mode humoristique qui moque les poncifs politiques et sociaux. Les énoncés décalent les expressions sources : annonce de la composition du gouvernement («IKEA ministre du Confort de l'intérieur»), assertion référendaire ou programmatique («Oui à la politique de détente», «Changeons tout de l’intérieur»), slogan politique ("La France réclame du neuf») ou social («Avis de rêve général»), pastiche de citation («Le design sera démocratique ou ne sera pas»). Un message télévisé privilégie dans le même temps le «besoin de changement», en offrant à la clientèle des solutions de rangement quotidien et de bien-être domestique : l'un des spots parodie un discours électoral et se termine parl'invite «Dites oui à une France dynamique. Votez IKEA» 27.

Les annonceurs tendent aujourd'hui à décliner le message commercial sur la longue période, exploitant les différentes séquences de l'élection, qui sert alors de fil conducteur à la communication. Les conséquences du vote et même le suivi des engagements électoraux sont pris en compte. IKEA revient ainsi ironiquement sur les résultats de 2007 et publie dans Le Monde du 8 mai une affiche représentant deux oreillers carrés de couleurs bleue et rouge, formant sur un fond blanc le drapeau tricolore, encadrés par le commentaire : «Sans regret... » [...] «Chez IKEA, vous disposez de 30 jours pour changer d'avis si vous pensez avoir fait le mauvais choix ${ }^{28}$. Les consommateurs ont effectivement cet avantage sur les électeurs.

Le vote passé, les publicitaires peuvent aussi saluer la fin du mandat des sortants (comme Canal +, avec son affiche «Pot de départ. Dimanche 6 mai

26. Cette thématique a été utilisée dès les législatives de 1993: « Le 28 mars, vous allez changer de chambre : profitez-en pour changer de literie » (cité par Albouy, 1994, p. 157, note 18).

27. Visuel : site 16 .

28. Site 17. 
à 20 h. Votez + », qui représente la marionnette emblématique de Jacques Chirac) ${ }^{29}$, ou inviter les électeurs à être attentifs aux promesses des élus (comme E. Leclerc) ou à dresser un bilan, comme la marque «Président », qui lance un spot télévisé pendant l'été 2007, à l'occasion du «bilan des cent jours » de Nicolas Sarkozy3o. Le site de voyages Lastminute.com exploite jusqu'aux débuts du mandat présidentiel : "Offrez-vous des vacances bling bling et augmentez votre pouvoir d’achat»; "Cet été, la France qui se lève tôt sera la première sur la plage» (Bénaroya, 2010).

- Mir (du groupe Henkel) avait déjà instrumentalisé le référendum sur Maastricht en 1992, en organisant pour le lancement d'un nouveau produit une consultation de la clientèle, avec l'accroche : «Quand on touche aux institutions, tout le monde a son mot à dire». La société réitère l'expérience en mars 2007, en privilégiant l'interactivité. Dans une campagne multicanal, elle présente au suffrage sept de ses produits phares, dont Mir Laine, qui déclare : «En votant pour moi, vous faites le choix de la tranquillité!». Exploitant «la crise de l'engagement » et l'émergence de la démocratie participative, l'annonceur inaugure l'«eMirlitantisme», nouvelle mouture de marketing participatif. Dans le cadre de l'opération "La France vote Mir», les internautes, stimulés par des cadeaux, peuvent mener campagne. L'enseigne, en jouant sur le principe communautaire et participatif par un système de parrainage et d'inscription qui rapporte des points, enrichit du même coup son fichier de clients ${ }^{31}$.

- La recherche d'implication du public, dans des simulacres (plus ou moins élaborés) de campagnes électorales, caractérise également l'initiative de la marque de lames de rasoirs Wilkinson en 2006, primée par la profession comme «la meilleure campagne interactive» (Grand prix Stratégies). En quête de distinction vis-à-vis de ses concurrents qui offrent des produits assurant un rasage toujours impeccable, Wilkinson ouvre un site de propagande (600 000 visiteurs) pour le Droit Aux Rasages Extravagants (DARE). Les affiches et vidéos reproduisent et tournent en dérision l'ambiance de la compétition électorale, en figurant les campagnes de trois grands candidats : «le free DARE», dont le slogan est : "Oui au poil libre»; «le DARE classic : Demi-barbes, grands hommes » et «le sexy DARE : les femmes votent sexy dare ». Usant d'un humour très décalé, la marque propose notamment aux adhérents de faux partis de monter leur film de campagne, de proposer leurs photos, en récompensant les participants, avant de soutenir en février 2007 la candidature présidentielle imaginaire de l’intègre médecin généraliste Pierre Hénaut32.

Quand ils n'inventent pas des candidats, les publicitaires se servent de

29. Affiche sur le site 9.

30. Sites 18 et 19.

31. Site 20.

32. Voir site 21. La série parodique «Hénaut Président», diffusée en 2007 sur Paris Première, sera reprise sous forme de long-métrage pour la présidentielle de 2012. 
l'offre électorale en présence, en transformant les faits et les acteurs33, sur un mode de plus en plus intrusif. Parmi les tendances apparues en $2007 \mathrm{se}$ détache la présence accrue d'arguments de séduction sexuelle. Ce phénomène s'explique sans doute par la présence d'une femme parmi les présidentiables et un probable et inédit duel de genre au second tour (Barbet, Mayaffre, 2009, p. 6), mais aussi par la tentation de la surenchère commerciale entre des marques et agences désireuses d'accrocher le public.

- Mentionnons à ce titre le visuel «viral» de Thomson pour le lancement d'un nouvel appareil GPS, conçue par Publicis-Net en novembre 200634. L'intrigue se déroule au soir du 6 mai 2007 (deuxième tour de la présidentielle), juste avant 20 heures : un homme ayant les traits de Nicolas Sarkozy quitte Neuilly en automobile avec son chauffeur, à qui il demande de se rendre «à l'Élysée»; deux minutes plus tard, une femme (la silhouette de Ségolène Royal) quitte précipitamment en vélo ce qui ressemble au siège du PS, dans le $7^{\mathrm{e}}$ arrondissement parisien, pour la même destination. Chaque véhicule est doté d'un GPS, qui conseille bien sûr au conducteur du premier de tourner à droite et à la cycliste d'aller à gauche... Les deux personnages se retrouvent finalement à 20 heures, à l'issue d'un parcours mouvementé, pour un baiser passionné et un rendez-vous amoureux à l'hôtel «Élysée »... Le message conclusif du film : « Ne vous fiez pas à votre première intuition. GPS Thomson-Intuiva. Il ne vous trompera pas », invite le spectateur à faire confiance à l'équipement plutôt qu'aux apparences de la lutte politique. La référence à l'adultère (polysémie de la tromperie) est évidemment activée par la notoriété des déboires conjugaux des deux protagonistes.

- Plus suggestive, la marque de lingerie Triumph International publie fin mars 2007 deux affiches de mannequins posant en dessous féminins 35 , placardées sur 12000 panneaux d'abribus, assorties de slogans évoquant de façon... à peine voilée, pour le premier, une invite... à la «participation» («Avec moi, pas d'abstention») et pour le second, le «soutien» («Enfin une candidature bien soutenue »), à l'heure où, précisément, la concurrente socialiste déplore d'en manquer de la part de ses camarades.

Les militantes féministes s'élèvent évidemment contre cette dévalorisation de l'image de la femme (quatre candidates sont alors en compétition), mais aussi contre la caricature du processus électoral, «ce temps fort de la démocratie »36. Sur le site de «la Meute des chiennes de garde contre la publicité sexiste ", plusieurs clientes annoncent même leur boycott de la marque. Pour sa part, le Bureau de Vérification de la Publicité37 rappelle dans un communiqué

33. Une vidéo caricaturale modifiant les noms des trois principaux candidats de 2007 , très stéréotypés, a été livrée sur Internet par une filiale des PagesJaunes (Les Échos, 12 avril 2007).

34. Visuel consultable sur le site 22. Le film a été vu plus d'un demi-million de fois sur Dailymotion dans le semestre précédant l'élection.

35. Reproduites sur le site 23.

36. "Le choix des (b)urnes », 9 avril 2007 : site 24.

37. Remplacé après 2007 par l'Autorité de régulation professionnelle de la publicité (ARPP). 
du 15 mai 2007 ses principes déontologiques en même temps que ses limites: il a permis, au «motif de la dignité des femmes », d'éviter un visuel encore plus provocant, mais n'a pas été suivi dans sa préconisation, fondée sur l'affirmation d'une réticence à «la référence au vote et à la politique en période électorale », de modifier les deux slogans. Outre que la profusion de cette référence l'expose à devoir exprimer désormais de nombreuses réserves, le BVP invoque en conclusion deux arguments peu susceptibles de réduire la Meute au silence : le premier apparemment juridique - l'absence de ressemblance physique avec une candidate en lice ne permet pas de «craindre un rapprochement douteux»-; le second, encore plus... "renversant»: "Aurait-on soupçonné une atteinte à l'image de leurs homologues masculins si la publicité avait représenté un homme? $» 38$

Autrement licencieux mais avec une audience plus limitée, un vendeur en ligne de sex toys exploite le fantasme de la femme dominatrice, en osant le message : «Pour la Saint-Nicolas, Ségolène a commandé des menottes... » (Héraud, 2007).

On se doute que certains des iconotextes, visuels ou propos mentionnés ci-dessus posent des problèmes de déontologie et de droit (respect du droit à l'image, de la vie privée des personnalités représentées, ou de leur fonction). Les professionnels de la publicité et les organismes régulant le secteur, tout en admettant que ces messages peuvent contribuer à la désacralisation politique, précisent parfois qu'ils la «regrettent» mais ne l'ont pas initiée, et s'attachent avant tout aux obstacles juridiques opposés au libre exercice de leur activité39. Bien sûr, la «peopolisation» des personnalités politiques les expose à une instrumentalisation promotionnelle par la publicité médiatique : RTL en février 2006 met ironiquement à l'affiche les «couples » HollandeRoyal et Sarkozy-Villepin sur le thème du «Vivre ensemble » et Europe 1 représente en septembre, avec un slogan plutôt racoleur - «Que faire pour que les jeunes croient à nouveau à la politique?»-, les mannequins rajeunis de François Bayrou, Nicolas Sarkozy, Ségolène Royal et Jack Lang. Au même moment, Laurent Fabius est pris au piège de son propre discours. Dans une interview à L'Express, il vient de mettre en avant son programme en soupçonnant implicitement sa concurrente à la primaire socialiste, Ségolène Royal, de céder au «peopolisme» : «Je préfère dire, a-t-il déclaré : "Voici mon projet”, plutôt que “mon projet, c'est Voici”. » Sitôt dit, le magazine à scandales Voici profite de l'aubaine en sortant une affiche qui ajoute à la phrase et au portrait de l'intéressé : «Quand Laurent Fabius nous fait de la pub, c'est Royal » $4^{\circ} \ldots$

De leur côté, les acteurs politiques ont jusque-là été peu enclins à se

38. Voir «Dessous et politique : fallait-il s'abstenir ? » : site 25.

39. Voir le site 8 (ARPP).

40. Voir le site 9. 
plaindre de la récupération publicitaire, qui peut aussi les servir. Il leur arrive parfois de protester. Par exemple, le Premier ministre Dominique de Villepin s'indigne en 2005 de l'affiche "Vivre ensemble» (avec Nicolas Sarkozy...) : " [Les hommes politiques n'ont] pas vocation à devenir des mannequins publicitaires, ni à faire de la publicité de quelque marque que ce soit» (février 2008). De même, après son instrumentalisation par Voici, Laurent Fabius réagit : «Je suis tout à fait opposé à la dérive people de la politique et de la presse, et cette utilisation faite à mon insu me renforce dans ma conviction. » 41 Les politiques se montrent plus consentants quand la cause est réputée «noble», comme pour la campagne dans la presse de la Mutualité française le 23 octobre 2007 : les deux «finalistes» de la présidentielle, avec le président de la MAAF, sont tour à tour mis en scène derrière un pupitre de meeting, avec la mention des effectifs de leurs publics : «19 millions de personnes» pour le premier, 17 pour la seconde, mais 38 millions de clients pour le président de la Mutualité. Non seulement les deux anciens candidats ont donné leur assentiment, mais leurs photographies ont été vendues à l'agence publicitaire42.

Ces emprunts de plus en plus marqués à la politique, pour vendre des marchandises, pourraient étonner, dans une conjoncture réputée "dépolitisée». Mais comme les humoristes, les publicitaires savent jouer sur plusieurs degrés et s'adresser à différents publics : à ceux qui continuent à être intéressés par les élections (assez nombreux en 2007), mais aussi à ceux qui expriment leur scepticisme ou leur dégoût de l'activité politicienne et sont susceptibles d'être séduits par la raillerie des propos. Leurs messages s'appuient sur une activité politique «en crise », la désaffection des citoyens facilitant sa mise en dérision. Ils privilégient la séduction dans la relation recherchée entre l'entreprise et ses clients, et accordent à ce titre une place décisive à l'humour par décalage. Ils s'apparentent à ce titre à la variante de publicité dite « oblique» (Lugrin, 2006, p. 171 et 194), fondée sur la connivence, articulée autour de l'ironie et des faits d'intertextualité (Charaudeau, Maingueneau, 2002, p.327-329). L'humour a pourtant des limites : si, dans les exemples étudiés, les «détrousseurs» de discours politiques s'amusent de la crise du militantisme, de l'offre électorale, des candidats, de leurs affiches, de leurs slogans, de leurs promesses, il est remarquable que le principe de l'élection et l'acte de vote sont eux-mêmes épargnés. Sans doute parce que ces derniers servent de paradigme pour le choix commercial escompté ("élire, comme acheter, c'est choisir»). Les annonceurs, préoccupés de voir leurs produits désignés par les consommateurs, ne

41. Ibid. pour les citations de Dominique de Villepin et Laurent Fabius.

42. Ibid. pour les différents visuels. La mutuelle d'assurances a proposé en 2007 un site électoral fictif (jelaurai. com), qui met en avant, pour une fois, un consommateur : son personnage fétiche Marcel Leraleur, candidat MAAF ( "Mouvement pour un Avenir Assurément Formidable »), doté d'une panoplie complète de campagne (Les Échos, 12 avril 2007 ; Héraud, 2007). 
sauraient remettre en cause la procédure élective. Mais ce respect est aussi le résultat de l'imposition de la doxa démocratique 43 : si les acteurs et leur «jeu » sont égratignés, le principe électif sort paradoxalement conforté par les usages commerciaux, le recours à l'électoral témoignant en définitive d'une extension du «vote» à des domaines toujours plus nombreux. Il est significatif, par exemple, qu'une campagne comme celle de Ben \& Jerry's, après que la vache Woody a passablement ridiculisé les mœurs politiques, se termine en 2007 par un appel au vote : le pseudo-cortège présidentiel de militants représentant l'enseigne se dirige en effet vers plusieurs bureaux de vote parisiens en «meuglant»: «Soyez pas vache, votez!»44.

Les risques de saturation et de banalisation auxquels s'exposent les parodies politiques pourraient les priver d'originalité et d'attractivité, ce qui serait un comble pour le marché publicitaire, mais celui-ci est connu pour ses capacités d'adaptation ou d'absorption. Certes, la transposition se révèle souvent assez pauvre, nombre de locuteurs ne disant pas beaucoup plus que «votez pour moi ». On pourra toutefois saluer, dans la masse inégalement subtile des discours, l'inventivité de certains «créatifs » et la sophistication de quelques métaphores filées. On ignore en fait dans quelle mesure ces procédés contribuent à l'augmentation des ventes. En tout cas, ils font réagir, que cette communication prête à sourire ou qu'elle agace le citoyen attaché à « une certaine image de la politique ». La pérennité et la multiplication de ces détournements politisants prouvent en tout cas que l'objet politique, même présenté sur un mode caricatural, est encore censé faire vendre. Bien sûr, il s'agit d'une conception réduite à la dimension électorale, cette dernière étant elle-même restreinte à une offre stéréotypée, à la rivalité et aux jeux interpersonnels, ce qui conforte la personnification et la spectacularisation. Mais cette vision repose sur - et traduit, à sa façon - celle que partage une large partie de l'opinion. Dans cette relation complexe et ambiguë45 à la politique, ces publicitaires contribuent en quelque sorte à la fabrication des tendances qu'ils dénoncent, pour mieux les exploiter. Tout en faisant mine parfois, avec une certaine hypocrisie, de les déplorer, ils font un peu penser à des pompiers incendiaires qui crieraient «A feu!»...

43. "L'évidence du vote tient enfin (surtout?) à son caractère doxique et aux tabous, voire aux interdits qui entourent sa dénaturalisation. L'équation élection = démocratie reste aujourd'hui irréfragable [...]» (Lehingue, 2011, p. 16).

44. Site 13.

45. Le «réinvestissement» (Maingueneau, 1991, p. 155) du genre politique dans ces messages publicitaires reste souvent ambigu, mélange de «captation » et de «subversion». 


\section{Liste des sites consultés}

1. [http://www.frontnational.com/videos/11-septembre-acropolis-nice\%E2\%80\%93-discours-de-marine-le-pen-videos/], c. 24 novembre 201146.

2. [http://www.lepost.fr/article/2010/03/10/1980593_votez-eliminez.html], c. 22 décembre 2011.

3. [http://www.lepost.fr/article/2011/01/07/2362631_avec-arnaud-montebourg-jepositive html], idem.

4. [http://www.ina.fr/economie-et-societe/environnement-et-urbanisme/video/ logo86606/nicolas-sarkozy-le-terme-nettoyer-au-karcher-est-un-terme-qui-simpose.fr.html], c. 14 juin 2008.

5. [http://lexpansion.lexpress.fr/entreprise/karcher-refuse-fadela-amara-enpublicitaire_224663.html], c. 16 février 2010.

6. [http://www.dailymotion.com/video/xaeugz_9o-ans-citroen_auto], 14 juin 2010

7. [http://www.ina.fr/pub/produits-d-entretien/video/PUB2346176013/eparcylfosse-tranquille-version-16-secondes.fr.html], c. 13 février 2009.

8. [http://www.arpp-pub.org/Recuperation-du-politique-en.html] [ octobre 2006], c. 14 avril 2011.

9. [http://www.politique.net/2008012701-media-quand-la-publicite-recupere-lespolitiques.htm], janvier 2008, c. 15 octobre 2009.

10. [http://niceplanneur.com/2011/11/23/vers-une-politisation-de-plus-en-pluspoussee-des-marques/], novembre 2011, c. 11 décembre 2011.

11. [http://www.les2vaches.com/notre-biographie/histoire-et-actions] et [http:// strategies.fr/actualites/marques/r91481W/deux-vaches-dans-la-campagne-presidentielle.html], c. 15 octobre 2011.

12. [http://www.blogoergosum.com/195-friskman-le-candidat-de-l-haleine-fraiche], c. 28 septembre 2011.

13. [http://www.e-marketing.fr/Breves/Ben-Jerry-s-part-en-campagne-20831.htm], c. 15 octobre 2011.

14. [http://img.over-blog.com/500×394/1/30/71/79/rep-5-sept-2009/Inter-40-ansprospectus.jpg], c. 28 septembre 2009.

15. [http://huguesdelabelliere.files.wordpress.com/2010/01/compterendu-37emesjourneesannuelles-matin2411.pdf], c. 14 juin 2010.

16. [http://www.dailymotion.com/video/k384TG2sRkorC88AKM\#from=embed], c. 3 février 2010 .

17. [http://www.strategies.fr/actualites/marques/r44694W/lendemain-electoralpour-ikea-et-president.html], idem.

18. [http://www.dailymotion.com/video/x2rg2h_pub-le-president_news], c. 22 août 2007.

19. [http://www.pointsdevente.fr/le-nouveau-president-revele-art71815-4.html], idem.

20. [http://www.prodimarques.com/sagas_marques/mir/mir.php], c. 31 janvier 2011.

21. [http://www.blogoergosum.com/230-les-marques-s-incrustent-dans-les-

46. «c.»+ date : date de la dernière consultation du site. 
presidentielles-episode-2-ikea-et-henkel], c. 3 février 2010.

22. [http://video.google.com/videoplay?docid=1700686196017346079], c. 29 septembre 2010.

23. [http://www.lapanse.com/pages/pubs/pub_du_jour/triumph_presidentielle_ no_abstention.html], c. 9 septembre 2010.

24. [http://www.lameute.fr/actualite/triumph.php3], c. 9 septembre 2010.

25. [http://www.arpp-pub.org/Dessous-et-politique-fallait-il-s.html] 15 mai 2007, c. 14 avril 2011.

\section{Références}

Albouy Serge, 2000 [1994], Marketing et communication politique, Paris, L'Harmattan (Logiques sociales).

BARBET Denis, 2007, "La politique est-elle footue? ", Mots. Les langages du politique, no 84, juillet, Politiquement sportif, p. 9-22.

Barbet Denis, Mayaffre Damon éd., 2009, Mots. Les langages du politique, no89, février, 2007. Débats pour l'Élysée.

BÉnAROYA Christophe, 2010, "L'engouement des marques pour "surfer" sur les élections. Le cas de la présidentielle», 4 pages, en ligne [www.christophe-benaroya.fr/ page22.php], consulté le 14 novembre 2011.

Bon homme Marc, 2006, «Parodie et publicité», Tranel, n44, p. 165-180.

CATHelat Bernard, Ebguy Robert, 1988, Styles de pub. 60 manières de communiquer. L'expérience Eurocom, Paris, Éditions d'Organisation.

Charaudeau Patrick, 2005, Le discours politique. Les masques du pouvoir, Paris, Vuibert.

Charaudeau Patrick, Maingueneau Dominique éd., 2002, Dictionnaire d'analyse du discours, Paris, Le Seuil.

Chauveau Agnès, Veyrat-Masson Isabelle, 2004, "L'histoire dans les spots publicitaires. Un mariage antinomique », Le Temps des médias, 1/2004, nº 2, p.127-136.

GRANIER Corinne, 2008, «La publicité politisée. Éloge de la transparence, ellipse de la responsabilité», Les enjeux de l'information et de la communication, nº 1/2008, p. 10-19.

HÉrAud Béatrice, 2007, «Détournements de campagne », Marketing Magazine, n¹13, p. 14-15.

Krieg-Planque Alice, 2009, La notion de "formule» en analyse de discours. Cadre théorique et méthodologique, Besançon, Presses universitaires de Franche-Comté.

LeHINGUE Patrick, 2011, Le vote. Approches sociologiques de l'institution et des comportements électoraux, Paris, La Découverte.

LUG RIN Gilles, 2006, Généricité et intertextualité dans le discours publicitaire de presse écrite, Berne, Peter Lang.

Maingueneau Dominique, 1991, L'analyse du discours. Introduction aux lectures de l'archive, Paris, Hachette.

Maudieu Marie, 2007, "La bonne pub à l'affiche», Stratégies Magazine, nº1469, 20 septembre.

SOUCHIER Emmanuël, 1992, "La publicité comme détournement du politique», Communication et langages, $\mathrm{n}^{\circ} 93, \mathrm{p} .36-51$. 